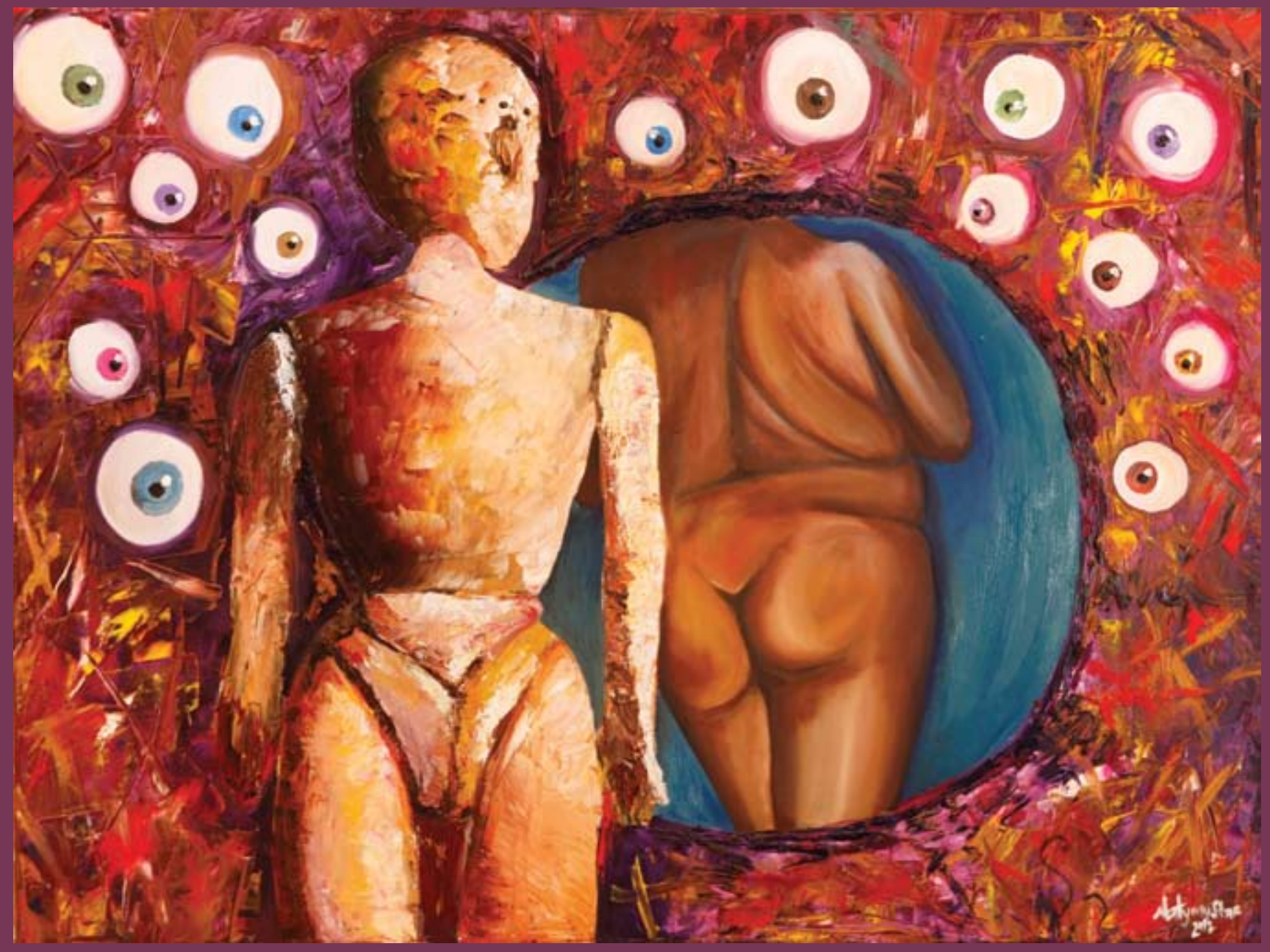

"Espejo Falso"

Oleo sobre Lienzo

Nataly Céspedes

Concurso ARTE + 2012

Bogotá - Colombia

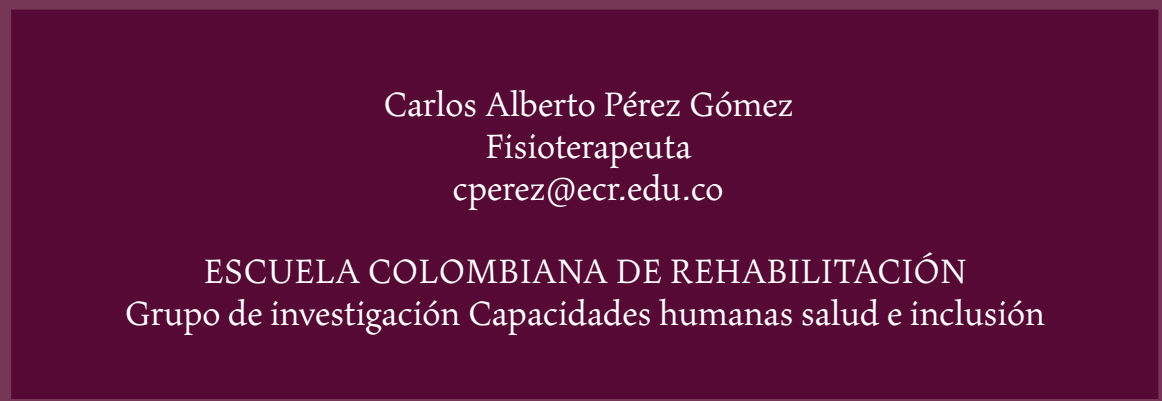

REV. COL. REH || Bogotá, Colombia || Volumen 11 || Página 80 - 86 || Noviembre 2012 || ISSN 1692 - 1879 


\title{
INSTRUMENTO PARA LA EVALUACIÓN DE LA PERCEPCIÓN DEL AMBIENTE COMO FACILITADOR O BARRERA EN PERSONAS CON EVENTO CEREBROVASCULAR
}

\section{Instrumentto assess the perception of the environmentas a facilitatoror barrierfor people who suffer from Cerebrovascular Disease}

Fecha de Recepción: 16 de Marzo de 2011 - Fecha de Aprobación: 3 de Octubre de 2011

\section{RESUMEN}

El Evento Cerebrovascular (ECV) ha sido reconocido como un objetivo importante de investigación desde diferentes áreas y enfoques de la salud. El ECV impacta el funcionamiento de la persona, y para su intervención se hace necesario contar con instrumentos de evaluación que logren reconocer sus efectos desde distintas dimensiones. Este artículo presenta el diseño de un instrumento para la evaluación de la percepción del ambiente (productos y tecnología; apoyo y relaciones) como facilitador o barrera en personas que han sufrido un ECV. Se realizó un estudio descriptivo de desarrollo tecnológico tomando como unidades de análisis la Clasificación Internacional del Funcionamiento de la Discapacidad y de la Salud (CIF) (OMS, 2001), e instrumentos realizados para la evaluación de Calidad de Vida relacionada con la salud. Se desarrolló un instrumento base, que fue sometido a validación de contenido. Se obtuvo un cuestionario con 75 preguntas que identifican percepción desde el ambiente como facilitador o barrera (no funcionalista), el cual fue sometido a validación por medio de jueces expertos que evaluaron la pertinencia de los ítems, y realizaron aportes a la construcción. Se calculó el índice de validez de contenido propuesto por Lashe y modificado por Tristán (2007), eliminando 8 ítems. Finalmente el estudio presenta un instrumento de 67 ítems distribuidos en 2 dominios y 16 subcategorías, beneficiando con esta herramienta a personas con secuelas de eventos neurológicos.

PALABRAS CLAVES

Evento cerebrovascular, CIF, Factores ambientales, apoyo y relaciones

\begin{abstract}
The Cerebrovascular Event (CVD) has been recognized as an important objective of research from different areas and approaches to health. The CVD impacts the person's performance, and for its intervention is necessary to have assessment tools that let recognize its effects from different dimensions. The proposing an instrument to assess environmental perceptions (products and technology, support and relationships) as a facilitator or barrier for people who have suffered from CVD. A descriptive-technological development study was performed. The International Classification of Functioning, Disability and Health (ICF) (OMS, 2001) were used as analysis units, as well as different instruments for the assessment of health related quality of life. A base instrument was developed which was submitted to content validation. A 75 questions questionnaire was developed to identify perception from the environment as facilitator or barrier (not functionalist), which was submitted to expert judges validation who evaluated the relevance of each item, and made contributions to the construction. Each item was submitted to content validity index that was proposed by Lashe and modified by Tristan (2007), thus, were removed of items, and the instrument was reduced to 67 items divided into two domains and 16 subcategories. This research generates an important contribution for decision-making beyond the clinic, and can also benefit people who have consequences of neurological events and can be extended later to other populations.
\end{abstract}

KEY WORDS

cerebrovascular disease, CIF, environmental factors, support and relationships 


\section{INTRODUCCIÓN}

L os sistemas de vigilancia epidemiológica en el mundo, así como investigaciones académicas han reportado a los eventos de tipo cerebrovascular como uno de los eventos de morbilidad y mortalidad con mayor frecuencia tanto a nivel internacional como nacional (Pradilla, Vesga, León, y grupo GENECO, 2003; OMS, 1989). Estudios en Colombia y en diferentes países de Latinoamérica encuentran coincidencias epidemiológicas en cuanto a los valores de incidencia que lo han llegado a posicionar en tercer y segundo lugar (respectivamente). El evento se encuentra asociado a condiciones relacionadas con la industrialización y al cambio en los ritmos de vida sociales, como el tabaquismo, las dietas no balanceadas, el sedentarismo, entre otros (ASCOFAME, 1998; Saposnik y Del Brutto, 2003; y Uribe, Arana y Lorenzana, 2000). Una vez se presenta el evento, se pueden presentar secuelas motoras, sensitivas y según el caso, cognitivas Wade (citado por Downie, 2008); estas secuelas dependerán de la zona cerebral afectada, de la extensión, y de condiciones socio-politico-demográficas que influyen sobre la persona. Ameriso y Esnaola, citados por Casas, Barreiro, Carmona y Rugilo (2005) mencionan que:

"Una tercera parte de los sobrevivientes requieren asistencia para las actividades de la vida diaria o para cuidado personal. El $16 \%$ de los pacientes permanecen hospitalizados por un periodo prolongado, el $20 \%$ necesitan de asistencia para caminar, y el 70\% no pueden regresar a su trabajo previo" (p.19)

La frecuencia e impacto del evento cerebrovascular lo han posicionado como una prioridad en salud pública, que genera además la necesidad de contar con instrumentos adecuados de evaluación no sólo de las estructuras y funciones, sino de las demás condiciones humanas que constituyen éste evento como una condición discapacitante. Mundialmente se han desarrollado estudios como los publicados por Grimby, Andre'n, Daving, \& Wright (1998); De Haan, Horn, Limburg, Van Der Meulen, \& Bossuyt, (1993); y Kwon, Hartzema, Duncan, \& Min-Lai (2004), que centran su interés en la evaluación realizada a las personas que han sufrido un ECV reconociendo las escalas que se emplean para ello; que se constituyen a partir de dominios que permiten evaluar el estado de las estructuras y el nivel de funcionamiento de las personas.

Así mismo, un estudio de Roberts \& Counsell (1998), desarrollado a partir de la revisión de 174 investigaciones analizadas en función de la Clasificación de deficiencias, discapacidades y minusvalías (CDDIM), menciona que el $76 \%$ y $42 \%$ de los documentos revisados midieron deficiencias o discapacidades respectivamente (bajo el modelo de discapacidad previo a la CIF), sólo unos pocos estudios evaluaron muerte o minusvalía (desventajas en la experiencia social), sin embargo, la evaluación se realizó de manera funcionalista. En este sentido, investigaciones de este tipo tienen en cuenta una perspectiva parcializada, siendo evidente que no se realiza un análisis desde cómo la persona interpreta aquello que en el contexto le facilita o que funciona como barrera para la realización de actividades o para participar en comunidad

Así mismo, cada vez son más frecuentes los estudios centrados en la percepción de la persona sobre sí mismo, tal es el caso de los estudios realizados hasta la fecha sobre Calidad de Vida Relacionada con la Salud (CVRS), en este sentido, Alonso (2000), presenta un argumento que soporta la comprensión parcial de éste fenómeno, cuando menciona que para la evaluación de la CVRS, se tienen en cuenta "ítems que reflejan el subconjunto de síntomas y limitaciones en las funciones física, psicológica y social que resultan más relevantes para dichos pacientes" (p, 165). Evidencia de ello es la investigación desarrollada por, Suárez-Escudero y cols (2011) denominada "Descripción clínica, social, laboral y de la percepción funcional individual en pacientes con ataque cerebrovascular", allí se emplean preguntas como: " $i$ Usted siente que ha mejorado la movilidad de su cuerpo o extremidad afectada tras el 'derrame y/o trombosis cerebral'?" para la descripción de la percepción funcional, (p.10o); haciendo evidente que se busca identificar la interpretación de la persona sobre sus propias limitaciones, no sobre los factores contextuales (externos a él).

Suárez Escudero y cols (2011) analizan la discapacidad asociada con el ECV como la consecuencia de diferentes factores y no como una simple "secuela" neurológica, determinando que los componentes ambientales representan una mayor barrera, que en sí la misma condición de salud o la deficiencia estructural o funcional de la cual padezca la persona. Argumentando que se deben implementar programas de seguimiento para poder identificar las necesidades de ésta población y se realicen actividades que aseguren una rehabilitación integral $y$ funcional.

Además de éstos estudios, se encuentran otros acercamientos a la evaluación de percepción con éste mismo alcance (Weimar et a, 2002; y Lai, Perera, Duncan, \& Bode, 2003; entre otros), que mantienen la misma postura sobre cómo la persona percibe las barreras o facilitadores que cierran o evitan el cierre del círculo discapacitante, sin embargo, el desarrollo de instrumentos de evaluación con ésta perspectiva es ausente.

A pesar de que las interpretaciones sobre la Clasificación internacional han llevado al abordaje tradicional centrado en el sujeto, han permitido dilucidar una serie de categorías (dominios) desde donde realizar análisis bajo un enfoque contextual no funcionalista. Por tanto, al momento de realizar un abordaje centrado en la percepción del sujeto alrededor de factores ambientales, debe tenerse en cuenta los aspectos más relevantes que permitan una adecuada articulación del análisis de los factores contextuales, con los análisis que se realizan desde el funcionamiento.

No sólo la mirada funcionalista genera preocupación en los instrumentos que actualmente existen para evaluación de percepción desde salud; los instrumentos mencionados anteriormente son aplicados sólo bajo condiciones de comunicación ora indemne, lo cual generó reflexiones importantes sobre la necesidad de realizar un proceso inclusivo independiente de las dificultades de comunicación oral, para fundamentar la necesidad de instrumentos que no excluyan por cuestiones fisiológicas como afasias de expresión, se localizó un estudio de gran relevancia realizado por Hilari, Byng, Lamping \& Smith (2003), quienes realizaron una apreciación muy relevante acerca de la valoración típica de la percepción del sujeto en términos de calidad de vida relacionada con la salud (CVRS) en personas con accidente cerebrovascular. Ellos mencionan que aunque existen diversas escalas tanto genéricas como específicas para Accidente Ce- 
rebrovascular, estas generalmente excluyen a las personas que tienen afasia o con desórdenes cognitivos, lo que hacía relevante contar con instrumentos que cuenten con las características clinimétricas que permitieran la evaluación de personas con esta condición. En función de estas apreciaciones, los autores diseñaron una escala denominada SAQOL-39 (stroke and Aphasia quality of life scale -39), que además de permitir la evaluación de calidad de vida específicamente en ECV, permite la evaluación en personas con afasia, o disfunciones cognitivas.

La SAQOL-79 identifica elementos relevantes en cuanto a la comunicación en personas que han sufrido un accidente cerebrovascular, y presenta dentro de su instrumento una serie de preguntas que buscan identificar hasta donde la persona percibe dificultades para expresar ideas y para ser entendido desde lo oral/verbal. Esta escala, aunque soluciona el inconveniente de aplicación a personas con afasia, sigue manteniendo un enfoque funcionalista.

Por otro lado, los investigadores cubanos Fernández y cols (2004), a partir de una problematización importante alrededor de la debilidad metodológica de algunos de los instrumentos actuales para la evaluación de CVRS, y además, ante la inexistencia documentada de escalas específicas en español, construyeron la Escala para Calidad de Vida en Ictus (ECVI 38) que a partir de un acuerdo de jueces (médicos expertos) identificaron 8 dominios bajo los cuales se construyó la escala, estos fueron: a) Problemas físicos, b) Comunicación, c) Cognición, d) Emociones, e) Sentimientos, f) Actividades básicas de la vida, g) Funciones familiares, y h) Funciones sociales. Esta escala demostró adecuados valores de validez y confiabilidad, a partir de la comparación con otras escalas internacionales, y a partir de evidencia de validez por aplicación a población de prueba. Esta prueba ha sido punto de referencia para la identificación del lenguaje pertinente para la elaboración de ítems a efectos de esta investigación.

Existe una característica que se considera relevante y ha sido evidenciada en los instrumentos específicos para ECV (en inglés y español), esta particularidad hace referencia a la mirada desde la cual se ha venido evaluando la percepción de salud.

Actualmente hay evidencia escrita que marca que cuando se realizan acciones en salud (etiológicas, diagnósticas o terapéuticas), es pertinente tener en cuenta la percepción de la persona como eje central de identificación de atributos como "calidad de vida relacionada con la salud", esto es conocido con el concepto de utilidad (Petrou, 200o), que se enfoca eminentemente en la valoración de los beneficios que proporciona una intervención en función de lo que la persona percibe como mejor. De esta forma, las investigaciones desarrolladas son abordadas pensando en la concepción del sujeto sobre sí mismo, quien desde sus deficiencias y limitaciones crea su percepción de salud. Este concepto no es necesariamente equivocado, dado que se basan en todo lo que la persona percibe acerca de ciertas características de su vida, sin embargo, esta percepción puede ser modificada por las condiciones fluctuantes o permanentes producidas por situaciones de discapacidad independientes a la persona; por lo cual se hace pertinente desarrollar estudios que permitan identificar la percepción del sujeto no sólo sobre sí mismo, sino sobre los aspectos externos que lo condicionan, reflexionando sobre cómo el ambiente participa en su situación/condición de salud en función de las facilidades y restricciones que genera.
Las investigaciones más recientes alrededor de la evaluación de personas con ECV, se han fundamentado en la Clasificación Internacional del Funcionamiento, de la Discapacidad y de la Salud (CIF), que ha funcionado como una plataforma para delimitar los aspectos a tener en cuenta al realizar aproximaciones desde la salud. En este sentido, para la Fisioterapia, articular el desarrollo de instrumentos desde una perspectiva mundial como la de la CIF logra la resonancia de los resultados y la interlocución a nivel internacional. Los estudios centrados en la CIF han abordado aspectos de estructuras, limitaciones en la realización de actividades y restricciones en la participación, sin embargo, los factores ambientales son delimitados por su gran relevancia dentro de la actual conceptualización de la salud y la discapacidad (Finger, Cieza, Stoll, Stucki, \& O Huber, 2006).

La clasificación internacional contempla dominios amplios dentro del ambiente, como son a) servicios, sistemas y políticas, b) actitudes, c) apoyo y relaciones, d) entorno natural y cambios en el entorno natural producto de la actividad humana, y e) productos $y$ tecnología; cada uno de estos factores implica una serie de categorías que en conjunto buscan identificar la totalidad de elementos externos al sujeto y que se constituyen en modificadores de la interacción y la forma de experimentar una deficiencia física. (OMS, 2001).

Bajo el paradigma de salud actual, que busca la interrelación entre el modelo biomédico y el modelo social, la discapacidad generada por un ECV debe entenderse más allá de la incapacidad para el desempeño (que centra la mirada únicamente en la persona). La interrelación de modelos permite aclarar que es indispensable tener en cuenta los factores contextuales que funcionan como barreras o facilitadores para la persona, siendo importante identificar si se tiene acceso o no a los recursos, si estos son de mala calidad, o si los factores de análisis se constituyen en una barrera, tanto por su presencia como por su ausencia. Estos factores contextuales son conceptualizados como "Factores Ambientales" en la Clasificación Internacional del Funcionamiento, de la discapacidad y la Salud (CIF) (OMS, 2001).

Ahora bien, la CIF menciona, que los factores ambientales "deben ser codificados desde la perspectiva de la persona cuya situación está siendo descrita" (OMS, 2001), esto específicamente está relacionado con las percepciones, es decir, con aquello que la persona reconoce e interpreta sobre su realidad, dado esto, la evaluación debe realizarse alrededor de lo que la persona percibe sobre los facilitadores o las barreras. Sin embargo, a pesar de esta importancia, este aspecto sólo se ha investigado desde una mirada de la función, sin poder identificar cómo las personas que han presentado un evento cerebrovascular interpretan sus condiciones ambientales y cómo éstas son generadoras de condiciones de discapacidad. Por tanto, no se conoce la perspectiva de la persona alrededor de las barreras o los facilitadores ambientales, las cuales quizá no son evidentes para una persona con estructuras indemnes y con un alto nivel de funcionamiento, pero para una persona que presenta secuelas de un ECV puede implicar la imposibilidad de satisfacer necesidades de salud, educación y ocio; así como a necesidades más básicas como el libre desenvolvimiento dentro del hogar.

Esta problemática lleva a plantear un estudio, que tiene como objetivo general realizar la caracterización de la percepción del apoyo 
y las relaciones, así como el acceso/utilización de productos y tecnología como facilitadores o barreras para el funcionamiento en personas con ECV. Sin embargo, ante la inexistencia de instrumentos de evaluación con este alcance, la primera parte de éste estudio, que genera el contenido del presente artículo corresponde al desarrollo de un instrumento que pretende reconocer los dominios que permiten documentar la percepción de las personas con ECV sobre barreras y facilitadores relacionados con el apoyo y relaciones, y los productos y tecnologías.

\section{MÉTODO}

El estudio realizado fue de tipo descriptivo en el cual se realizó un proceso de desarrollo tecnológico orientado a delimitar un instrumento para la evaluación de la percepción del ambiente por parte de personas que han sufrido un evento cerebrovascular. Esta investigación se orienta al desarrollo de un producto tangible, de forma que se pueda identificar su evidencia de validez. El manual Frascati publicado por la Organización para la cooperación y el desarrollo económico (OCDE), menciona que los estudios con éste alcance son "trabajos sistemáticos fundamentados en los conocimientos existentes obtenidos por la investigación o la experiencia práctica, que se dirigen a la fabricación de nuevos materiales, productos o dispositivos, a establecer nuevos procedimientos, sistemas y servicios, o a mejorar considerablemente los que ya existen" (OCDE, 2003). El proceso se realizó bajo un diseño de corte transversal basado en los aspectos metodológicos de la Clinimetría, considerada como "una disciplina centrada en el desarrollo y la validación de las evaluaciones clínicas". Se ha venido consolidando como un área interdisciplinar soportada por las ciencias médicas, la psicología y la estadística (Tomba y Fava, 2006). Permite identificar elementos de validez de una prueba de evaluación de aspectos clínicos. Se utilizó como unidad de análisis, la clasificación Internacional del Funcionamiento, la discapacidad y la Salud. A partir de las categorías del apartado de Ambiente, se generaron los dominios y reactivos para la evaluación de los componentes de productos y tecnología y apoyo y relaciones que desde la perspectiva CIF llevan a la evaluación del contexto desde la perspectiva de la persona. El instrumento desarrollado fue sometido a un proceso de validación de contenido, aplcando el índice de validez de contenido desarrollado por Lawshe en 1977 y modificado por Tristán en 2007 (Tristán y Molgado, 2007).

\section{RESULTADOS}

Se elaboró un instrumento de evaluación basado en las categorías actualmente existentes en la Clasificación. Se generaron preguntas específicas en cada categoría y opciones de respuesta basadas en escala likert, sin embargo, dada la novedad del instrumento, se abrió un espacio para respuesta libre por parte de las personas, que puede dar origen a nuevas categorías para la conformación definitiva del instrumento.

Las categorías de los apartados "Productos y Tecnología", y "Apoyos y Relaciones" de la CIF que se tuvieron en cuenta para la propuesta de instrumento fueron tomadas de forma cercana a la estructura del documento de CIF del 2001, y a partir de éstas categorías se generó la estructura de abordaje, de forma que permitiera iden- tificar la percepción de la persona tanto de su acceso, como de las barreras y facilitadores que genera el medio para dicho acceso.

Los factores ambientales son calificados como facilitadores o barreras, entendiendo que un elemento ambiental funciona como facilitador en la medida de la calidad del recurso, y en la medida en que el medio favorece la accesibilidad de forma constante o variable al recurso. Ahora bien, el elemento funciona como barrera en la medida que sea un obstáculo ya sea por a) su presencia, b) por su ausencia, c) por la frecuencia en que es un "estorbo", d) su tamaño y, e) la posibilidad de evitarlo.

En éste sentido, dada la amplitud de categorías de la CIF en cuanto a los factores contextuales, las categorías seleccionadas para ésta investigación fueron "productos y tecnología", y "apoyo y relaciones".

En cuanto a productos y tecnología, la CIF los define como: "Cualquier producto, instrumento, equipo, o tecnología adaptada o diseñada específicamente para mejorar el funcionamiento de una persona con discapacidad" (p. 187).

En cuanto a apoyo y relaciones, la CIF menciona que éste apartado "Trata sobre las personas (y animales) que proporcionan apoyo, educación, protección, asistencia y relaciones con otras personas, en sus casas, en sus lugares de trabajo, en la escuela o en el juego o en cualquier otro aspecto de sus actividades diarias. El capítulo no abarca las actitudes de la persona o personas que proporcionan el apoyo. El factor ambiental descrito no hace referencia a la persona o al animal sino a la cantidad de apoyo físico y emocional que la persona o el animal proporciona".

Una vez delimitado el alcance del instrumento se desarrolló un instrumento de evaluación de 75 ítems manteniendo las categorías de productos y tecnología, y apoyo y relaciones propuestas en la CIF, 53 ítems correspondieron al dominio productos y tecnología, y 22 correspondieron a dominio apoyo y relaciones.

El instrumento diseñado fue enviado a cuatro (4) evaluadores, tres conceptuales y uno metodológico. Los evaluadores conceptuales con experiencia en discapacidad y con conocimiento de la clasificación internacional del funcionamiento, la discapacidad y la salud (CIF), se escogió un número impar de evaluadores conceptuales para tener criterios de desempate ante los desacuerdos posibles. Para la elección del evaluador metodológico se tuvo en cuenta la experiencia en el diseño y evaluación de instrumentos de evaluación, con formación en medición y evaluación. La evaluación representaba dos momentos, el primero buscó identificar acuerdos y desacuerdos entre los jueces, y el segundo permitió la incorporación de observaciones realizadas por los evaluadores con intención de ajustar el contenido y la forma.

Los instrumentos evaluados, fueron sistematizados para así aplicar el índice de validez de contenido (CVR) propuesto por Lashe (1977), sin embargo, este índice requiere para su precisión un número mayor de jueces al empleado en éste estudio, por tanto, una vez obtenido el índice, se aplicó modelo de normalización (CVR') propuesto por Tristán (2007), que permite lograr mayor precisión 
con un número limitado de jueces, estableciendo un punto de corte en 0.5823 , a partir del cual se asume que un ítem fue evaluado como pertinente (figura 1 y 2 ).

$$
C V R=\frac{n_{e}-\frac{N}{2}}{\frac{N}{2}}
$$

Figura 1. Índice de validez de contenido, Lashe (1977)

$$
C V R^{\prime}=\frac{C V R+1}{2}
$$

Figura 2. Índice adaptado por Tristán, (2007)

La sistematización arrojó un resultado de 9 ítems con puntajes en el índice normalizado menores de 0,5823 (ver tabla 1), los cuales fueron eliminados del instrumento.

\begin{tabular}{|c|c|c|l|c|c|c|}
\hline Ítem & CVR & CVR' & & Ítem & CVR & CVR' \\
\hline 1 y 2 & 0,3333 & 0,6667 & & 31 a 33 & 1,0000 & 1,0000 \\
\hline 3 & $-0,3333$ & 0,3333 & & 34 & $-0,3333$ & 0,3333 \\
\hline 4 & $-0,3333$ & 0,3333 & & 35 a 56 & 0,3333 & 0,6667 \\
\hline 5 & $-0,3333$ & 0,3333 & & 57 & $-0,3333$ & 0,3333 \\
\hline 6 & $-0,3333$ & 0,3333 & & 58 a 60 & 0,3333 & 0,6667 \\
\hline 7 y 8 & 0,3333 & 0,6667 & & 61 & $-0,3333$ & 0,3333 \\
\hline 9 & $-0,3333$ & 0,3333 & & 62 a 64 & 0,3333 & 0,6667 \\
\hline 10 a 25 & 0,3333 & 0,6667 & & 65 & $-0,3333$ & 0,3333 \\
\hline 26 a 29 & 1,0000 & 1,0000 & & 66 a 75 & 0,3333 & 0,6667 \\
\hline 30 & 0,3333 & 0,6667 & & & & \\
\hline
\end{tabular}

Tabla 1. Indice de validez de contenido e indice normalizado.

Los ítems restantes que incluían observaciones de contenido y forma fueron ajustados uno a uno, lo que permitió el desarrollo de un instrumento final con 2 dominios (apoyo y relaciones; $y$ productos y tecnología), 16 categorías (Alimentación; medicamentos; productos de tecnología generales para uso personal en la vida diaria; productos y tecnología generales para la movilidad y el transporte personal en espacios cerrados y abiertos; productos y tecnología para la comunicación; productos y tecnología de ayuda para la educación; productos y tecnología de ayuda para el empleo; productos y tecnología de ayuda para las actividades recreativas y deportivas; productos y tecnología de ayuda para la práctica de la religión y la espiritualidad; productos y tecnología relacionados con la arquitectura; la construcción y el diseño de edificios para uso público; familiares cercanos; amigos; conocidos, compañeros, colegas, vecinos y miembros de la comunidad; cuidadores y personal de ayuda; profesionales de la salud; y extraños), y 67 ítems. La estructura de preguntas para cada dimensión se encuentra en las tablas 2 y 3.

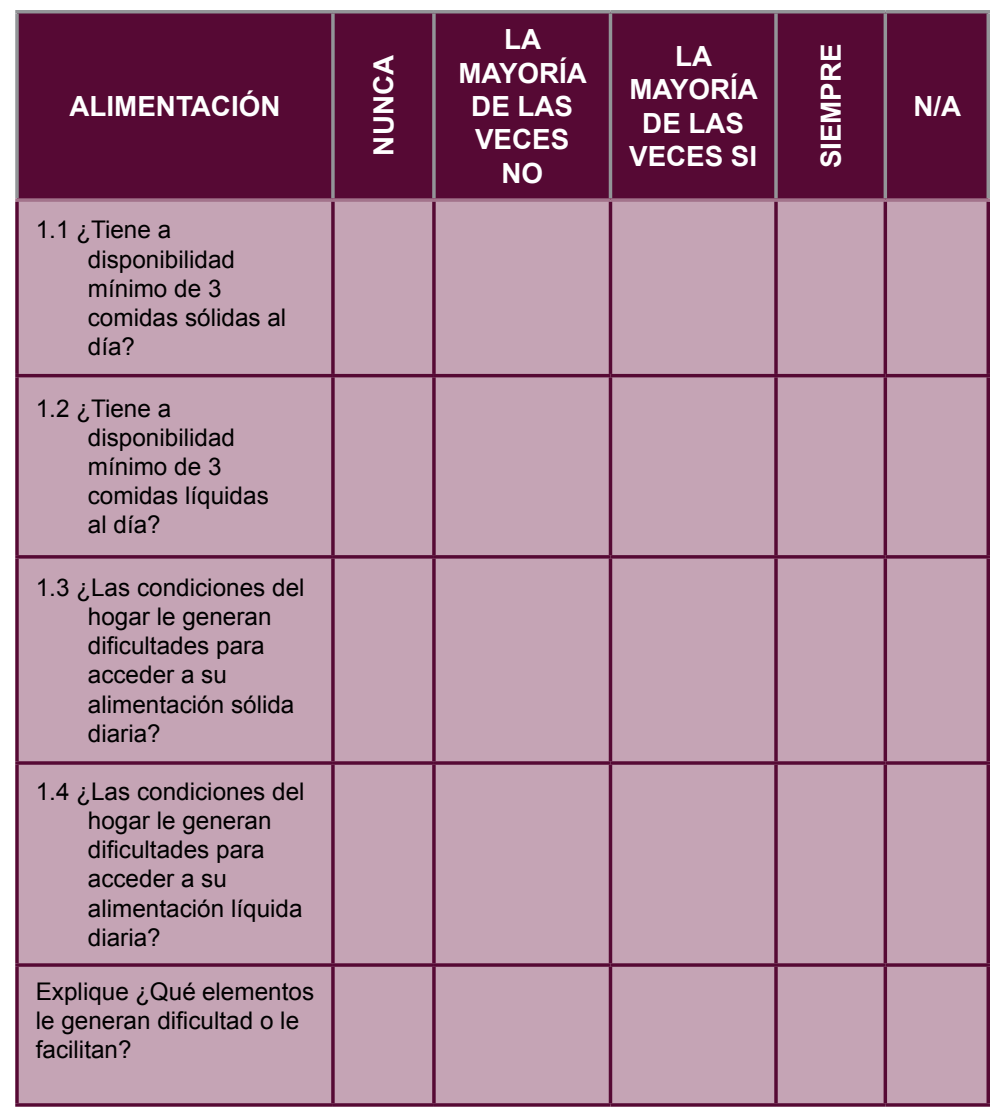

Tabla 2. Modelo de preguntas dimensión productos y tecnología

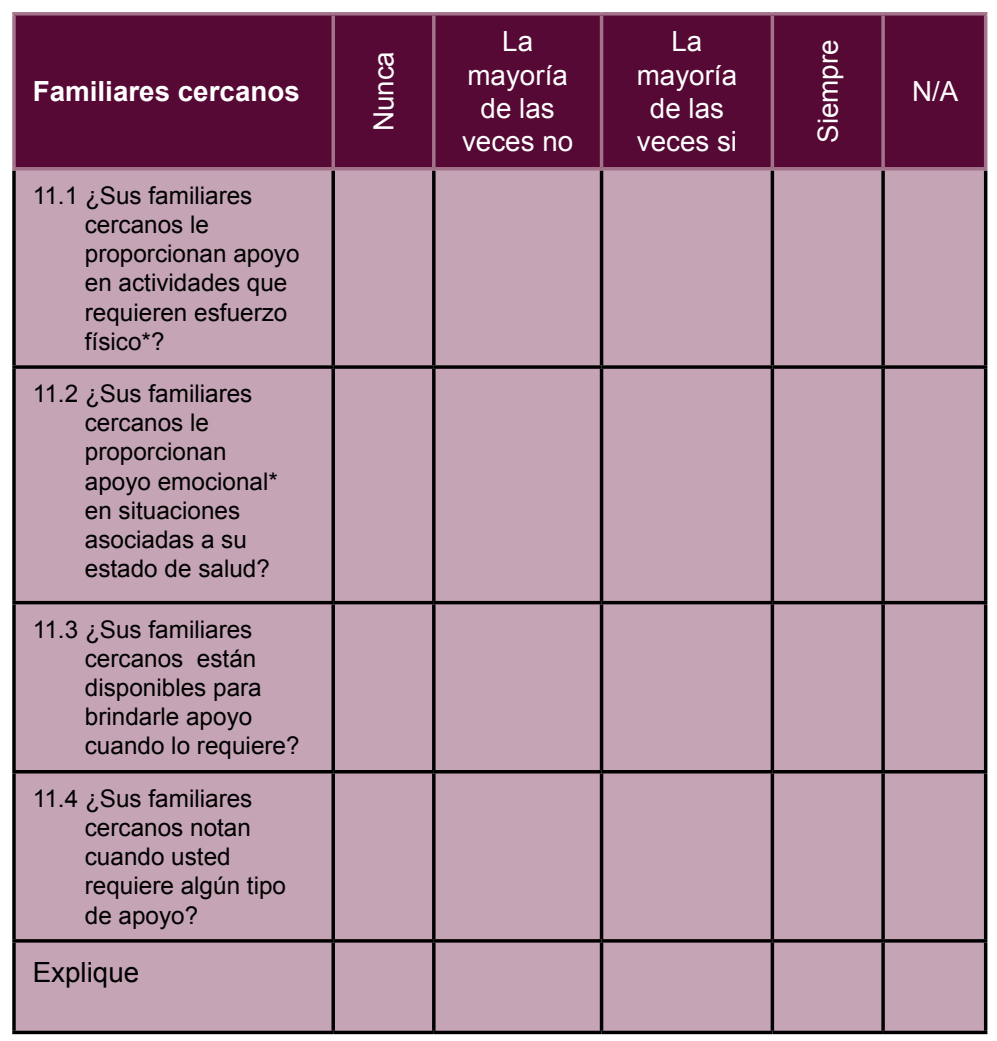

Tabla 3. Modelo de preguntas dimensión apoyo y relaciones 
9 CONCLUSIONES

La generación de instrumentos de evaluación implica un proceso de evolución permanente que debe responder a la necesidad de reconocer las particularidades de las personas, a las diferencias de las diferentes condiciones de salud, y a las tendencias teóricas y los modelos de intervención que tanto a nivel nacional como internacional se encuentran vigentes. Este instrumento requiere mayor desarrollo aplicado que permita obtener la información necesaria para realizar un análisis factorial conducente a un instrumento con mayor precisión.

A partir del proceso adelantado se tiene plena confianza sobre su utilidad práctica en la identificación de elementos importantes relacionados con las condiciones potencialmente discapacitantes que aún no han sido registradas desde la perspectiva de la persona con ECV debidas a la ausencia de instrumentos.

\section{Referencia:}

\section{Las referencias a otras obras son una parte}

muy importante en la literatura científica; ya que estas permiten conocer más sobre los autores y mantener vivas sus voces dentro del texto.

1. Alonso, J. (2000).La medida de la calidad de vida relacionada con la salud en la investigación y la práctica clínica. Gaceta Sanitaria. 14 (2):163-167.

2. Ascofame (1998). Guías de práctica Clinica basadas en la Evidencia. Enfermedad Cerebrovascular. Asociación Colombiana de Facultades de Medicina.

3. Casas, I.; Barreiro, L.; Carmona, S.; y Rugilo, C. (2005). Manual de Neurología. Segunda edición. Colombia: Grupo editorial Guía.

4. De Haan, R.; Horn, J.; Limburg, M.; Van Der Meulen, J.; \& Bossuyt, P. (1993). A Comparison of Five Stroke Scales With Measures of Disability, Handicap, and Quality of Life. Stroke. 24:1178-1181

5. Downie, P. (2008). CASH. Neurología para Fisioterapeutas, Cuarta edición. Buenos Aires, Argentina: Editorial Médica Panamericana.

6. Fernández-Concepción, O. et al (2004). Desarrollo de una escala para evaluar la calidad de vida en los supervivientes a un Ictus. Revísta de Neurología. 39 (10): $915-923$.

7. Finger, M.; Cieza, A.; Stoll, J.; Stucki, G.; y O Huber E. (2006). Identification of Intervention Categories for Physical Therapy, Based on the International Classification of Functioning, Disability and Health: A Delphi Exercise Physical Therapy . Volume 86 . Number 9 . September 2006

8. Grimby, G.; Andre'n, E.; Daving, Y.; Wright, B. (1998). Dependence and Perceived Difficulty in Daily Activities in Community-Living Stroke Survivors 2 Years After Stroke A Study of Instrumental Structures. Stroke; 29;1843-1849

9. Hilari, K.; Byng, Lamping, \& Smith (2003).Stroke and Aphasia Quality of Life Scale-39 (SAQOL-39).Evaluation of Acceptability, Reliability, and Validity. Stroke AHA. $1944-1950$.

10. Kwon, S.; Hartzema, A.; Duncan, W. \& Min-Lai S. (2004). Disability Measures in Stroke: Relationship Among the Barthel Index, the Functional Independence Measure, and the Modified Rankin Scale. Stroke. 35;918-923.

11. Lai, S.; Perera, S.; Duncan, D. \& Bode, B. (2003). Physical and Social Functioning After Stroke: Comparison of the Stroke Impact Scale and Short Form-36. Stroke. 34;488-493
12. Organización para la cooperación y el desarrollo económico OCDE OCDE (2003). Manual de Frascati. Propuesta de Norma Práctica para Encuestas de Investigación y Desarrollo Experimental.

13. Organización Mundial de la salud (1989). Recommendations on Stroke Prevention, Diagnosis, and Therapy.Stroke. 20; 1407-1431

14. Organización Mundial de la salud (2001). Clasificación Internacional de Funcionalidad, de la discapacidad y de la Salud. Grupo de Clasificación, Evaluación, Encuestas y Terminología: Ginebra, Suiza.

15. Petrou, S. (2000).What are health utilities?.Evidence Based Medicine. Vol 1 No 4.Aventis pharma.

16. Pradilla, G.; Vesga, A.; León, F. y grupo GENECO (2003) Estudio neuro-epidemiológico nacional. (EPINEURO) colombiano. Revista Panamericana de Salud Publica. 14(2).

17. Robert, L. \& Counsell, C. (1998). Assessment of Clinical Outcomes in Acute Stroke Trials. Stroke, 29:986-991

18. Saposnik, G.; Del Brutto, O. (2003). Stroke in South America: A Systematic Review of Incidence, Prevalence, and Stroke Subtypes. Stroke 34;2103-2107

19. Suárez-Escudero, J.; Restrepo-Cano, S.; Ramírez, E.;Bedoya, C.; Jiménez, I. (2011). Descripción clínica, social, laboral y de la percepción funcional individual en pacientes con ataque cerebrovascular. Acta Neurológica Colombiana. Vol 27, (2): 97-105.

20. Tomba di E \& Fava G (2006). L'approccio clinimetrico in psicologia clinica. Rivista di Psicologia Clinica n.2/3. 141-151.

21. Tristán, A. y Molgado, D. (2007). Tablas de Validez de Contenido. Instituto de Evaluación e Ingeniería Avanzada, S.C. Sal Luis de Potosí. México

22. Uribe, C.; Arana, A.; y Lorenzana, P. (2000). Fundamentos de Medicina:Neurología. Quinta edición. Medellín, Colombia: Corporación para Investigaciones Biológicas.

23. Weimar, Ch.; Kurth, T.; Kraywinkel, K.; Wagner, M.; Busse, O.; Ludwig Haberl, R.; \& Diener, H. (2002).Assessment of Functioning and Disability After Ischemic Stroke. Stroke; 33;2053-2059. 